\title{
The study of prompt fission $\gamma$ rays at the Oslo Cyclotron Laboratory
}

D. Gjestvang ${ }^{1, *}$, S. Siem ${ }^{1}$, F. Zeiser ${ }^{1}$, J. Randrup ${ }^{2}$, R. Vogt ${ }^{3,4}$, F. Bello-Garrote ${ }^{1}$, L.A. Bernstein $^{2,5}$, D.L. Bleuel ${ }^{3}$, M. Guttormsen ${ }^{1}$, A. Görgen ${ }^{1}$, A.C. Larsen ${ }^{1}$, K.L. Malatji ${ }^{6,7}$, E. Matthews ${ }^{5}$, V. Modamio ${ }^{1}$, A. Oberstedt ${ }^{8,9}$, S. Oberstedt ${ }^{10}$, T. Tornyi ${ }^{11}$, G. Tveten ${ }^{1}$, A. Voyles ${ }^{5}$, and J. Wilson ${ }^{12}$

${ }^{1}$ Department of Physics, University of Oslo, Oslo, Norway

${ }^{2}$ Nuclear Science Division, LBNL, Berkeley, USA

${ }^{3}$ Nuclear and Chemical Sciences Division, LLNL, Livermore, USA

${ }^{4}$ Physics Department, University of California, Davis, USA

${ }^{5}$ Nuclear Engineering Department, University of California, Berkeley, USA

${ }^{6}$ iThemba LABS, Somerset West, South Africa

${ }^{7}$ Physics Department, University of Stellenbosch, Matieland, South Africa

${ }^{8}$ ELI-NP/IFIN-HH, Bucharest-Magurele, Romania

${ }^{9}$ Department of Physics and Astronomy, Uppsala University, Sweden

${ }^{10}$ European Commission, Joint Research Center for Nuclear Safety and Security, Geel, Belgium

${ }^{11}$ MTA-ATOMKI, Debrecen, Hungary

${ }^{12}$ IPN Orsay, Orsay Cedex, France

\begin{abstract}
The study of prompt fission $\gamma$ rays (PFGs) is crucial for understanding the energy and angular momentum distribution in fission, and over the last decade there has been an revived interest in this aspect of fission. We present the new experimental setup at the Oslo Cyclotron Laboratory for detecting PFGs resulting from charged particle-induced fission. Additionally, PFGs from the reaction ${ }^{240} \mathrm{Pu}(\mathrm{d}$,pf) were measured in April 2018, and the fission gated proton- $\gamma$ coincidence spectrum is shown. In order to explore the dependence of the PFG emission on the excitation energy and angular momentum of the compound nucleus, we plan several experiments where charged particle reactions are used to induce fission in various plutonium isotopes. The final results will be compared to predictions made by the Fission Reaction Event Yield Algorithm (FREYA) in an upcoming publication, to benchmark the current modelling of both the PFGs and the fission process.
\end{abstract}

\section{Introduction}

In 2019 we celebrate 80 years since the discovery of fission, but still a lot of properties of fission are not quite understood. One of these aspects is the emission of prompt fission $\gamma$ rays (PFGs) which are photons emitted from the fission fragments as they de-excite. These photons carry valuable information about the fissioning system [1], and by examining them, useful insights can be obtained as we try to understand how fission unfolds. Furthermore,

\footnotetext{
*e-mail: dorthea.gjestvang @ fys.uio.no
} 
a detailed understanding of PFG emission is crucial for reactor applications. The prompt photons, though carrying only a small percentage of the total energy released in fission [2], might deposit energy far from where they are emitted. Thus, good knowledge of the PFGs is essential when designing the next generation of nuclear reactors. Especially the fast neutron region is important, where the PFG characteristics are the least studied [3].

The prompt fission $\gamma$ rays are characterised by their photon spectrum (PFGS), from which the quantities the average total energy released per fission $E_{\text {tot }}$, the average multiplicity per fission $M_{\mathrm{g}}$, the average photon energy $E_{\mathrm{g}}$, can be extracted. Over the last decade, several measurements of these PFG characteristics have been conducted, see for example Refs. [3-6], and the focus has been on determining PFGs from neutron-induced fission of key actinides. Theoretical efforts have also been made to model PFG emission [7-9]. By comparing model predictions to experimental measurements, we can benchmark the current modelling of photon emission from fission.

In order to get a broader perspective on the behaviour of the PFGs, the Oslo Cyclotron Laboratory (OCL) has started a campaign to measure the PFGs resulting from charged particle-induced fission, which has several advantages compared to neutron-induced fission. The energy of the charged projectile can easily span a range of energies, and by detecting the energy of the ejectile the excitation energy $E_{\mathrm{x}}$ of the fissioning nucleus can be determined. Charged particle-induced fission thus enables us to inspect the PFG dependence on $E_{\mathrm{x}}$. Furthermore, surrogate reactions with charged particles are expected to induce more angular momentum in the compound nucleus [10]. Thus, by comparing PFG characteristics measured through different surrogate reactions, their dependence on angular momentum can be explored. The OCL experimental setup combines a charged particle detector, fission detectors, and a state-of-the-art $\mathrm{LaBr}_{3}$ scintillator detector array which makes it a powerful tool for studying prompt fission $\gamma$ rays through charged particle-induced fission.

\section{Experimental setup for PFG detection at the OCL}

The Oslo Cyclotron Laboratory has an MC-35 Scanditronix cyclotron which can deliver a pulsed beam of protons, deuterons, ${ }^{3} \mathrm{He}$, or $\alpha$ particles. The charged particle beam is steered to the experimental hall, where it induces various reactions in the target. When studying prompt fission $\gamma$ rays, fission events are selected using fission fragment detectors that are PPACs (Parallel Plate Avalanche Counter). These are gas-filled, low pressure detectors sensitive to heavy ions only [11], and are placed $5 \mathrm{~cm}$ in the forward direction, see figure 1 . The placement of the PPACs is important, as the fission fragments are easily stopped and cannot travel through the supporting backing of the targets. For the same reason, the reaction chamber must be in vacuum. The ejectile from the charged particle reaction is detected by the silicon detector ring SiRi, placed in the backward position. SiRi consists of 64 particle telescopes with a front-thickness of $130 \mu \mathrm{m}$ and back-thickness of $1550 \mu \mathrm{m}$ [12], and by observing the energy and angle of the ejectile, the compound nucleus excitation energy is reconstructed.

The prompt fission $\gamma$ rays are detected by the Oslo SCintillator ARray (OSCAR), consisting of 30 inorganic cerium-doped $\mathrm{LaBr}_{3}$ scintillator detectors. The detectors are cylinders, and each measures $88.9 \mathrm{~mm} \times 203 \mathrm{~mm}$ (diameter $\times$ length). The energy resolution of these detectors is about twice as good as the resolution for $\mathrm{NaI}(\mathrm{Tl})$ detectors, with $<2.5 \%$ resolution for $1.3 \mathrm{MeV} \gamma$ rays. The decay time is also about one-tenth of the decay time of $\mathrm{NaI}(\mathrm{Tl})$. As the previous photon detector array (CACTUS) at the OCL consisted of $\mathrm{NaI}(\mathrm{Tl})$ detectors, this is a major improvement in both the time and energy resolutions, and opens for new experiments to be performed. In figure 1 the layout of the experimental setup is illustrated. 


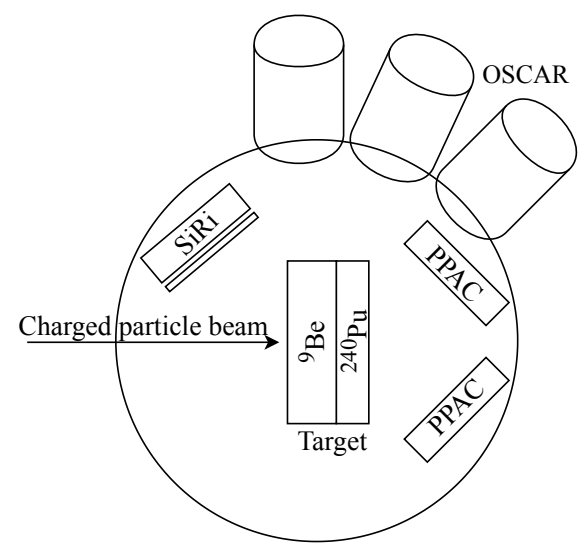

Figure 1. Experimental setup for detecting PFGs at the OCL, illustrated with the target used in the April 2018 experiment. Three of the $30 \mathrm{LaBr}_{3}$ detectors forming OSCAR are shown.

\subsection{The ${ }^{240} \mathrm{Pu}(\mathrm{d}, \mathrm{pf})$ experiment}

The first PFG experiment using OSCAR was run in April 2018. A target of $0.4 \mathrm{mg} / \mathrm{cm}^{2}$ thick ${ }^{240} \mathrm{Pu}$, placed on a backing of $2.3 \mathrm{mg} / \mathrm{cm}^{2}{ }^{9} \mathrm{Be}$, was bombarded with $13.5 \mathrm{MeV}$ deuterons and the resulting PFGs were measured. The distance between OSCAR and the target was chosen to be $20 \mathrm{~cm}$ for most of the detectors. The majority of the prompt fission neutrons, which can create photon-like signals in the $\mathrm{LaBr}_{3}$ detectors, could be rejected through the time-of-flight technique. One detector was pulled back to $40 \mathrm{~cm}$ to further test the ability to discriminate between neutrons and $\gamma$ rays. At this time, 28 of the $30 \mathrm{LaBr}_{3}$ detectors were installed.

\section{Results}

The data analysis for the ${ }^{240} \mathrm{Pu}(\mathrm{d}, \mathrm{pf})$ experiment is ongoing and will be published in an upcoming paper. Figure 2 shows the raw data matrix, with the number of $\gamma$ rays in coincidence with both a fission fragment and a proton plotted as a function of compound nucleus excitation energy $E_{\mathrm{x}}$ and measured photon energy $E_{\gamma}$. Note that $E_{\gamma}$ represents the measured energy of a photon and is different from $E_{\mathrm{g}}$ which is the average PFG energy. At the conference, the preliminary PFG photon spectrum was shown, plotted for different ${ }^{241} \mathrm{Pu}$ excitation energy bins. The photon spectrum was compared to predictions made with FREYA (Fission Reaction Event Yield Algorithm) which provides a complete description of fission where all physical quantities are conserved [13]. Preliminary extracted values for $E_{\mathrm{tot}}, M_{\mathrm{g}}$, and $E_{\mathrm{g}}$ were shown, plotted as a function of $E_{\mathrm{x}}$.

In order to obtain the true photon spectrum of the PFGs, the measured spectrum must be unfolded using the detector response function. The detector response function of OSCAR is not yet properly determined for photon energies below $500 \mathrm{keV}$. This is unfortunate, as the low-energy photons have been shown to have a large effect on the determined PFG characteristics [14]. To correct this, measurement of the PFGS from the spontaneous fission of ${ }^{252} \mathrm{Cf}$ is planned. As the PFGS from ${ }^{252} \mathrm{Cf}(\mathrm{sf})$ is well known, it acts as a benchmark for the experimental setup and the data analysis routine. 


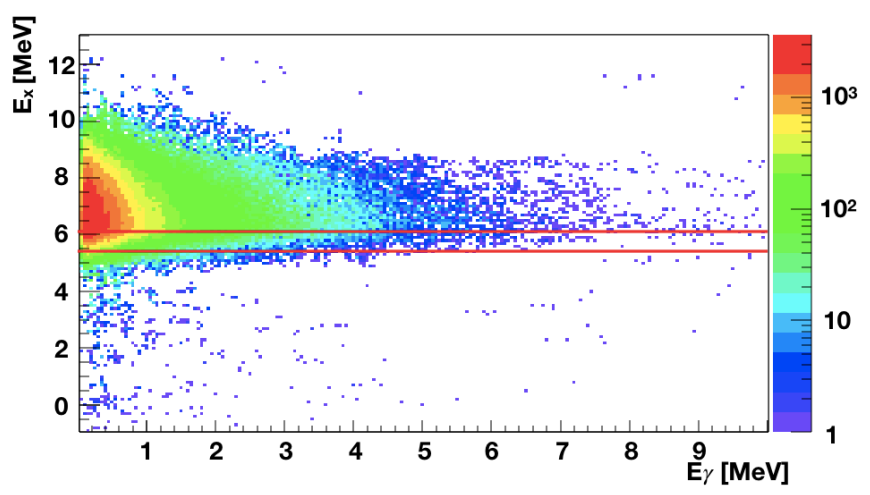

Figure 2. The coincidence matrix, showing the energies $E_{\gamma}$ of $\gamma$ rays emitted from ${ }^{240} \mathrm{Pu}(\mathrm{d}$,pf) events on the $x$-axis, plotted against the excitation energy $E_{\mathrm{x}}$ of ${ }^{241} \mathrm{Pu}^{*}$ on the $y$-axis. The colors on the right-hand side of the plot show the number of photons in each $\left(E_{\gamma}, E_{\mathrm{x}}\right)$ bin. The red lines represent the values for the double-humped fission barrier, as reported in Ref. [15, pg. 800].

\section{Outlook}

A range of PFG experiments are scheduled to run at the OCL with the new experimental setup that includes OSCAR. In the spring of 2020, PFG characteristics will be extracted from the ${ }^{239} \mathrm{Pu}(\mathrm{d}, \mathrm{pf})$ reaction. The $\mathrm{PFG}$ resulting from ${ }^{239} \mathrm{Pu}(\mathrm{n}, \mathrm{f})$ have been measured multiple times $[4,16-18]$, and (d,pf) is a surrogate reaction for (n,f). Even though both reactions produce the compound nucleus ${ }^{240} \mathrm{Pu}^{*},(\mathrm{~d}, \mathrm{pf})$ is expected to impart more angular momentum to the fissioning nucleus, allowing us to study the unknown impact of additional angular momentum on the PFG characteristics.

${ }^{239} \mathrm{Pu}(\mathrm{d}, \mathrm{pf})$ has previously been run at the OCL and results are published by Rose et al. in Ref. [10]. However, the old photon detector array CACTUS was used. The time resolution of these $\mathrm{NaI}$ detectors did not allow for a separation between prompt neutrons and photons through TOF, and the photon detection threshold was $400 \mathrm{keV}$. Therefore, we repeat the experiment to both obtain PFG characteristics below $400 \mathrm{keV}$ and to improve the experimental results above this threshold.

Another experiment, scheduled for spring 2020, is to induce fission in a ${ }^{244} \mathrm{Pu}$ target through a variety of charged particle reactions. This allows for the production of exotic actinides and studying their PFGs will further broaden our understanding of photon emission from the fission fragments.

Thus, we plan to study prompt fission $\gamma$ rays over a wide range of compound nucleus excitation energies and angular momenta. Through new experiments, and comparisons between experimental results and FREYA predictions, we expect to gain new insights into the behaviour of prompt fission $\gamma$ rays and therefore also the fission process.

The authors would like to thank J. C. Möller, P. Sorbas, and J. C. Wikne at the Oslo Cyclotron Laboratory for providing the high-quality deuteron beam during the experiment. This work was supported by the Research Council of Norway under project Grant No. 263030, and under the auspices of the U.S. Department of Energy by Lawrence Livermore National Laboratory under Contract DE-AC52- 
07NA27344. A. C. L. gratefully acknowledges funding through ERC-STG-2014 under grant agreement no. 637686. The work of R.V. was performed under the auspices of the U.S. Department of Energy by Lawrence Livermore National Laboratory under Contract DE-AC52-07NA27344. The work of J.R. was performed under the auspices of the U.S. Department of Energy by Lawrence Berkeley National Laboratory under Contract DE-AC02-05CH11231. This work was supported by the Office of Defense Nuclear Nonproliferation Research \& Development (DNN R\&D), National Nuclear Security Administration, U.S. Department of Energy.

\section{References}

[1] D. Choudhury, A. Gatera, A. Göök, M. Lebois, A. Oberstedt, S. Oberstedt, L. Qi, J. Wilson, AIP Conf. Proc. 2076, 060002 (2019)

[2] G. Rimpault, D. Bernard, D. Blanchet, C. Vaglio-Gaudard, S. Ravaux, A. Santamarina, Phys. Procedia 31, 3 (2012)

[3] L. Qi, M. Lebois, J.N. Wilson, A. Chatillon, S. Courtin, G. Fruet, G. Georgiev, D.G. Jenkins, B. Laurent, L. Le Meur et al., Phys. Rev. C 98, 014612 (2018)

[4] A. Gatera, T. Belgya, W. Geerts, A. Göök, F.J. Hambsch, M. Lebois, B. Maróti, A. Moens, A. Oberstedt, S. Oberstedt et al., Phys. Rev. C 95, 1 (2017)

[5] A. Oberstedt, T. Belgya, R. Billnert, R. Borcea, T. Brys̀, W. Geerts, A. Göök, F.J. Hambsch, Z. Kis, T. Martinez et al., Phys. Rev. C 87, 051602(R) (2013)

[6] M. Lebois, J.N. Wilson, P. Halipré, A. Oberstedt, S. Oberstedt, P. Marini, C. Schmitt, S.J. Rose, S. Siem, M. Fallot et al., Phys. Rev. C 92, 034618 (2015)

[7] R. Vogt, J. Randrup, Phys. Rev. C 96, 064620 (2017)

[8] I. Stetcu, P. Talou, T. Kawano, M. Jandel, Phys. Rev. C 90, 024617 (2014)

[9] M.J. Marcath, R.C. Haight, R. Vogt, M. Devlin, P. Talou, I. Stetcu, J. Randrup, P.F. Schuster, S.D. Clarke, S.A. Pozzi, Phys. Rev. C 97, 044622 (2018)

[10] S.J. Rose, F. Zeiser, J.N. Wilson, A. Oberstedt, S. Oberstedt, S. Siem, G.M. Tveten, L.A. Bernstein, D.L. Bleuel, J.A. Brown et al., Phys. Rev. C 96, 014601 (2017)

[11] T. Tornyi, A. Görgen, M. Guttormsen, A. Larsen, S. Siem, A. Krasznahorkay, L. Csige, Nucl. Instrum. Meth. A 738, 6 (2014)

[12] M. Guttormsen, T.E. Hansen, N. Lietaer, Nucl. Instrum. Meth. A 648, 168 (2011)

[13] J.M. Verbeke, J. Randrup, R. Vogt, Comput. Phys. Commun. 222, 263 (2018)

[14] A. Oberstedt, R. Billnert, F.J. Hambsch, S. Oberstedt, Phys. Rev. C 92, 014618 (2015)

[15] S. Bjørnholm, J.E. Lynn, Rev. Mod. Phys. 52 (1980)

[16] V.V. Verbinski, H. Weber, R.E. Sund, Phys. Rev. C 7, 1173 (1973)

[17] A. Chyzh, C.Y. Wu, E. Kwan, R.A. Henderson, T.A. Bredeweg, R.C. Haight, A.C. Hayes-Sterbenz, H.Y. Lee, J.M. O’Donnell, J.L. Ullmann, Phys. Rev. C 90, 14602 (2014)

[18] F. Pleasonton, Nucl. Phys. A 213, 413 (1973) 